\title{
Review
}

\section{The neuroprotective mechanism of brain ischemic preconditioning}

\author{
Xiao-qian LIU, Rui SHENG, Zheng-hong QIN \\ Department of Pharmacology and Laboratory of Aging and Nervous Diseases, Soochow University School of Medicine, Suzhou \\ 215123, China
}

\begin{abstract}
Brain ischemia is one of the most common causes of death and the leading cause of adult disability in the world. Brain ischemic preconditioning (BIP) refers to a transient, sublethal ischemia which results in tolerance to later, otherwise lethal, cerebral ischemia. Many attempts have been made to understand the molecular and cellular mechanisms underlying the neuroprotection offered by ischemic preconditioning. Many studies have shown that neuroprotective mechanisms may involve a series of molecular regulatory pathways including activation of the $\mathrm{N}$-methyl- $D$-aspartate (NMDA) and adenosine receptors; activation of intracellular signaling pathways such as mitogen activated protein kinases (MAPK) and other protein kinases; upregulation of Bcl-2 and heat shock proteins (HSPs); and activation of the ubiquitin-proteasome pathway and the autophagic-lysosomal pathway. A better understanding of the processes that lead to cell death after stroke as well as of the endogenous neuroprotective mechanisms by which BIP protects against brain ischemic insults could help to develop new therapeutic strategies for this devastating neurological disease. The purpose of the present review is to summarize the neuroprotective mechanisms of BIP and to discuss the possibility of mimicking ischemic preconditioning as a new strategy for preventive treatment of ischemia.
\end{abstract}

Keywords: brain ischemia; brain ischemic preconditioning; NMDA receptors; mitogen-activated protein kinases; heat shock proteins; reactive oxygen species

Acta Pharmacologica Sinica (2009) 30: 1071-1080; doi: 10.1038/aps.2009.105; published online 20 July 2009

\section{Introduction}

Generally speaking, any stimulus capable of causing injury to a tissue or organ can, when applied close to (but below) the threshold of damage, activate endogenous protective mechanisms and thus potentially lessen the impact of a subsequent, more severe attack. A sub-threshold ischemic insult applied to an organ, for example, activates cellular pathways that can help to reduce damage caused by subsequent severe ischemic episodes - a phenomenon known as ischemic preconditioning (IP) or ischemic tolerance (IT) ${ }^{[1]}$. This observation suggests that ischemic preconditioning is an adaptive reaction to a potentially noxious stimulus, such as ischemia, hypoxia, hypoglycemia, or inflammation. Although tolerance to ischemic insults can also result from cortical spreading depression ${ }^{[2]}$, sleep deprivation ${ }^{[3]}$, dietary restriction ${ }^{[4]}$, metabolic inhibition and oxygen free radicals ${ }^{[5]}$, and both hyperthermia and hypothermia ${ }^{[1]}$, ischemic preconditioning has been more aggressively studied.

\footnotetext{
* To whom correspondence should be addressed.

E-mail Zhqin5@hotmail.com

Received 2009-03-05 Accepted 2009-05-25
}

Ischemic preconditioning was first identified in the heart but was subsequently found to occur in the brain as well, where it is known as BIP. The brain is one of the most sensitive organs to injury. It is well accepted that, at least in mammals, the brain is approximately $3 \%$ of the body mass but receives approximately $15 \%$ of the total cardiac output and consumes $20 \%$ of the body's oxygen. A constant flow of blood to the brain is essential for delivering oxygen and glucose to neurons. If this flow is disrupted for even a short period of time, the result is cell damage or death. Neurons are rarely replaced once they have died, so the damage to affected regions may be permanent ${ }^{[6]}$. It has been found that there are two threshold values for cerebral blood flow. Reduction in blood flow below the first threshold results in electrical failure within neurons, and further reduction in blood flow below the second threshold leads to the failure of metabolism and ion pumps. Cells with lower perfusion than the second threshold are designated to die. Between the two thresholds, cells are electrically silent but maintain a low level of metabolic activity and can be stable for hours. If normal blood flow is restored within a reasonable amount of time, they may recover with no apparent damage. Longer periods of ischemia, however, will result in their 
death. So these cells have a variable fate, and constitute what is known as the ischemic penumbra ${ }^{[1]}$. It is the cells within the penumbra that receive the most benefit from ischemic preconditioning, as their chance for survival is increased through mechanisms evoked by BIP.

In recent years, the mechanisms of BIP have been systematically studied and several molecular regulatory pathways participating in preconditioning have been discovered. This opens a window to uncover endogenous neuroprotective mechanisms and, potentially, a window of opportunity to utilize these mechanisms in the clinic to treat patients with stroke and other CNS disorders. This review surveys the current understanding of cerebral preconditioning and the neuroprotective mechanisms evoked by BIP.

\section{Excitatory/inhibitory neurotransmitters and neuro- protection}

\section{Glutamate and NMDA receptors}

Glutamate is the major excitatory neurotransmitter in the mammalian brain and a key mediator of intercellular communication, neuronal plasticity, development, and differentiation of neurons. Under normal physiological conditions, the extracellular concentration of glutamate is maintained in the micromolar range and is responsible for initiation of postsynaptic excitatory signaling through distinct ionotropic and metabotropic glutamate receptors. The ionotropic glutamate receptors include the $N$-methyl- $D$-aspartic acid (NMDA) receptor, the 2-amino-3-(3-hydroxy-5-methylisoxazol-4-yl) proprionate (AMPA) receptor, and the kainate receptor subtypes ${ }^{[7]}$. Glutamate has long been known for its capability to kill neurons through the NMDA receptor-mediated mechanism, and NMDA receptor antagonists have been shown to be neuroprotective in animal models of several neurological disorders. Unfortunately, many NMDA receptor antagonists have undesirable side effects in humans that make them unsuitable for therapeutic use.

Paradoxically, subtoxic concentrations of NMDA protect neurons against glutamate-mediated excitotoxicity ${ }^{[8]}$. Bond et al reported that the non-competitive NMDA receptor antagonist MK-801 and the competitive NMDA receptor antagonist LY202157 all produced significant reductions in the induced ischemic tolerance, suggesting that mild NMDA receptor activation is involved in the neuroprotective mechanism of $\mathrm{BIP}^{[9]}$. Consistent with these findings, exposure of cortical cell cultures to low levels of glutamate or NMDA to induce NMDA receptor activation has a preconditioning effect ${ }^{[10]}$.

One underlying mechanism involved in the NMDA receptor-mediated BIP is the rapid adaptation of the voltagedependent calcium flux. Shimazaki ${ }^{[11]}$ showed that the hippocampus of gerbils that did not acquire tolerance showed a high elevation of calcium flux, while tolerant gerbils were better able to regulate calcium and kept calcium below the critical level for initiating neuronal death. Since hypoxia/ischemiainduced neurodegeneration can be triggered by cytoplasmic calcium overload, the NMDA receptor mediates rapid calcium adaption in preconditioning that may alleviate the cell damage caused by calcium overload.

The other mechanism of NMDA receptor neuroprotection involves the activation of NMDA receptors leading to the rapid release of brain-derived neurotrophic factor (BDNF). $\mathrm{BDNF}$ then binds to and activates its cognate tyrosine kinase B (TrkB) receptors. Both NMDA and TrkB receptors activate nuclear factor kappaB (NF-kB), a transcription factor which is expressed in neurons. NF- $\mathrm{kB}$ is activated in response to a variety of stress- and injury-related stimuli including exposure to cytokines such as tumor necrosis factor- $\alpha$ (TNF- $\mathrm{a})$, and excitotoxic and oxidative insults. Although NF-kB is an important inflammatory factor often involved in neuronal injury, it may play a part in the anti-death actions of TNF-a in cultured hippocampal neurons exposed to metabolic and oxidative insults. Mattson $e t a l^{[12]}$ reported that activation of NF-KB protected hippocampal neurons against oxidative stress-induced apoptosis, suggesting that NF-KB is involved in protecting neurons under certain conditions.

Other key mediators which have been involved in synaptic NMDA receptor-dependent neuroprotection include cyclic AMP responsive element binding protein (CERB), phosphatidylinositol 3 (PI3)-kinase, Akt and glycogen synthase kinase 3 beta (GSK3 $\beta$ ). These mediators can be induced only by low doses of NMDA via the action potential-dependent route ${ }^{[13,14]}$. CREB is a transcription factor that is activated in response to intracellular calcium elevation regulated by NMDA receptor activation ${ }^{[15]}$. Phosphorylation of CREB is rapidly and robustly enhanced in the penumbral region of preconditioned rats ${ }^{[16]}$, and thus is implicated as an important player in the preconditioning process mediated by NMDA receptor.

\section{GABA and GABA receptors}

Gamma-aminobutyric acid (GABA) is a well-known inhibitory neurotransmitter in the brain. Recently, Dave et al found that BIP promoted a robust release of GABA in rats after lethal ischemia ${ }^{[17,18]}$. They also observed that the activity of glutamate decarboxylase (the rate-limiting enzyme in GABA synthesis in the brain) was higher in the BIP group compared with controls and ischemic groups. They further tested the hypothesis that $\mathrm{GABA}_{\mathrm{B}}$ receptor activation was also neuroprotective during ischemia or early reperfusion by using an in vitro model (organotypic hippocampal slice culture). They found that administration of the $\mathrm{GABA}_{\mathrm{B}}$ agonist baclofen during ischemia and the first hour of reperfusion provided significant neuroprotection. They concluded that increased GABA release in preconditioned rats after ischemia might be one of the factors responsible for $\mathrm{BIP}$ neuroprotection and that $\mathrm{GABA}_{B}$ receptors may be the GABA receptors activated after BIP. In addition, Sommer's group has shown that ischemic tolerance in the preconditioned gerbil hippocampus is associated with increased ligand binding to inhibitory $\mathrm{GABA}_{\mathrm{A}}$ receptors between $30 \mathrm{~min}$ and $48 \mathrm{~h}$ of recirculation ${ }^{[19]}$. GABAergic disinhibition has been attributed to lesion progression because neuronal hyperexcitability associated with a sustained downregulation of $\mathrm{GABA}_{\mathrm{A}}$ receptors was found in peri-infarct regions. Preconditioning can cause increased GABA release or increased GABA recep- 
tor expression. The changes in GABAergic transmission, both pre- and postsynaptic, are likely to contribute to a shift of the glutamate/GABA balance toward inhibition in the preconditioning brain ${ }^{[20]}$.

\section{Adenosine and $\mathrm{K}^{+}$channels}

In 1995, Heurteaux et al demonstrated the essential role of adenosine, adenosine A1 receptors, and ATP-sensitive $\mathrm{K}^{+}$ channels in BIP. Adenosine is an endogenous neuroprotectant that can inhibit the release of excitatory amino acids. When ischemia occurs, adenosine can increase conspicuously. Adenosine inhibits synaptic transmission, decreases $\mathrm{K}^{+}$-stimulated glutamate release, and inhibits presynaptic calcium fluxes via adenosine A1 receptors. The response to calcium influx is also important for the development of protection against ischemia, because calcium influx has been linked to the production of reactive oxygen species and the initiation of a number of signaling cascades leading to cell damage ${ }^{[21]}$. Many reports have shown that preconditioning-induced neuroprotection is dependent on adenosine A1 receptors. In rats, ischemic preconditioning increased adenosine A1 receptor immunoreactivity in the hippocampal CA1 region at days 1, 3, and 7 after preconditioning induction, within the window of ischemic tolerance $^{[22]}$. Some unspecific and specific adenosine A1 receptor antagonists abolished the ischemic tolerance. Moreover, Liu et al also demonstrated that isoflurane-induced tolerance against focal cerebral ischemia in the rat middle cerebral artery occlusion (MCAO) model was attenuated by adenosine A1 receptor antagonists ${ }^{[23]}$.

Activation of the ATP-sensitive potassium channels $\left(\mathrm{K}^{+}{ }_{\text {ATP }}\right.$ channels) also has a role in BIP. Blockade of the $\mathrm{K}_{\text {ATP }}^{+}$channels abolished preconditioning and the protection afforded by adenosine and R-PIA (an adenosine A1 receptor agonist). By contrast, a $\mathrm{K}^{+}{ }_{\text {ATP }}$ channel opener (RP-52891, aprikalim) induced ischemic tolerance. Meanwhile, recent evidence showed that transient infusion of the $\mathrm{K}^{+}{ }_{\text {ATP }}$ channel antagonist sulfonylurea tolbutamide prior to ischemia could block BIP protection after forebrain ischemia, whereas pinacidil, a $\mathrm{K}^{+}{ }_{\text {ATP }}$ channel agonist, can emulate BIP in hippocampal slices ${ }^{[24]}$. Although the precise $\mathrm{K}_{\text {ATP }}^{+}$channels involved in BIP remain undefined, two $\mathrm{K}_{\text {ATP }}^{+}$channels have been described recently. One of these channels resides in the mitochondrial inner membranes; the other resides in the plasma membranes. The mitochondrial $\mathrm{K}_{\text {ATP }}^{+}\left(\mathrm{mK}^{+}{ }_{\text {ATP }}\right)$ has been suggested to be the key channel involved in ischemic preconditioning, because the $\mathrm{mK}^{+}{ }_{\text {ATP }}$ blocker 5-hydorxydecanoate (5-HD) prevented BIP-induced neuroprotection. It has been hypothesized that opening the $\mathrm{mK}^{+}{ }_{\text {ATP }}$ channels may depolarize mitochondrial membrane potential and promote an increase in the electron transport chain rate and thus increase ATP production ${ }^{[25]}$.

\section{Opioid receptors}

There are three types of opioid receptor: $\delta, \kappa, \mu$ opioid receptors. The activation of opioid receptors is neuroprotective when the body encounters ischemia, hypoxia and cold. Zhang and collaborators showed that the $\delta$ opioid receptors were involved in hypoxia preconditioning in cultured rat cortical neurons ${ }^{[26]}$. They observed that $\delta$ opioid receptor activation protected cortical neurons from hypoxia injury, whereas the $\delta$ opioid receptor antagonist naloxone blocked such protection. In addition, Zhao et al found that morphine (an agonist for $\delta, \kappa, \mu$ opioid receptors) and Tan-67 (a selective $\delta$ receptor agonist) induced a delayed preconditioning effect both in vivo and in vitro. The morphine preconditioning-induced neuroprotection was inhibited by $\beta$-funaltrexamine, a $\mu$-opioid receptor antagonist, but not by 7-benzylidenenaltrexone, a $\delta$-receptor antagonist, or norbinaltorphimine, a k-receptor antagonist. The Tan- 67 preconditioning-induced neuroprotection was inhibited by 7-benzylidenenaltrexone. These results suggest that the delayed phase of morphine preconditioning may involve $\mu$ opioid receptors and $\delta$ opioid receptors. Morphine and Tan-67 may activate a shared intracellular signaling pathway to induce the delayed preconditioning effects in the brain $^{[27]}$

\section{Inflammatory cytokines}

Inflammatory cytokines are known to have an important role in acute stroke. Cytokines such as interleukin-1 (IL-1), and TNF- $\alpha$ are important mediators of the inflammatory reactions seen in cerebral ischemia. The importance of the cytokine system in the setting of ischemia was emphasized by Kariko et $a l^{[28]}$. TNF-a is one of the pro-inflammatory cytokines and is expressed in the ischemic brain. Nawashiro et al ${ }^{[29]}$ studied the effects of pretreatment with TNF administered intracisternally in mice that were subjected to MCAO $48 \mathrm{~h}$ later. A significant reduction in infarct size was noted in mice pretreated with TNF at the dose of 0.5 microgram/mouse. Immunohistochemical analysis of brains subjected to $24 \mathrm{~h}$ of MCAO revealed a significant decrease in CD11b immunoreactivity after TNF pretreatment compared with the MCAO control. Therefore, TNF induces significant protection against ischemic brain injury and is likely to be involved in the signaling pathways that regulate ischemic tolerance. Liu et al ${ }^{[30]}$ demonstrated that preconditioning of rat cortical neurons with mild hypoxia protected them from hypoxia and oxygen and glucose deprivation (OGD) injury $24 \mathrm{~h}$ later (50\% protection). Interestingly, TNF-a pretreatment could be substituted for hypoxic preconditioning (HP). HP was attenuated by TNF-a-neutralizing antibody. Ohtsuki et $a l^{[31]}$ explained the role of IL-1 in the induction of tolerance to global ischemia in Mongolian gerbils. Arterial IL-1 $\alpha$ and IL-1 $\beta$ became elevated between 1 and 3 days after a 2-min ischemic exposure. Recombinant human IL-1 receptor antagonist (IL-1ra) ip blocked ischemic tolerance induction by 2-min preconditioning ischemia. The possible mechanisms of IL-1 action include release of arachidonic acid, enhancement of NMDA activation and stimulation of nitric oxide synthase ${ }^{[32]}$.

\section{Intracellular survival signals and neuroprotection MAPK, Akt/PKB, and PKC signal pathways}

In ischemic conditions, rapid changes occur in the activity of many different signaling paths, involving diverse protein kinase families. Alterations in the expression or activity of 
MAPK, Akt/protein kinase B (PKB), and protein kinase C (PKC) suggest that multiple kinases participate in the response to ischemia and reperfusion. MAPK-mediated signaling participates in the transduction of cellular responses from the extracellular environment to the nucleus and other intracellular targets, through sequential phosphorylation. The activation of specific components of MAPK cascades involves conserved three kinase modules consisting of MAPK, MAPK kinase (MEK), and MAPK kinase kinase (MEKK). It is now becoming evident that MAPK signaling plays a significant part in cerebral ischemia ${ }^{[33,34]}$. Neuronal apoptosis and cerebral ischemia both induce the robust activation of MAPK cascades. However, Zheng et al also demonstrated that preconditioninginduced neuroprotection against ischemia was mediated by activation of MAPK signaling pathway including extracellular signal-regulated kinases (ERK), c-Jun N-terminal protein kinases and $\mathrm{p} 38^{[35,36]}$. MAPKs are activated by phosphorylation on both threonine and tyrosine residues and subsequently phosphorylate intracellular enzymes and transcription factors. Generally speaking, ERK promotes cell survival and proliferation, whereas c-Jun N-terminal protein kinases induce apoptosis. Therefore, the roles of MAPK cascades in neuronal death and survival seem to be complex and can be altered by the types of cells and the magnitude and timing of insults.

$\mathrm{Akt} / \mathrm{PKB}$ is a serine/threonine kinase primarily involved in cellular survival pathways by inhibiting apoptotic processes. Akt can also induce protein synthesis pathways, and is therefore a key signaling protein in the cellular pathways that lead to skeletal muscle hypertrophy and general tissue growth. Since it can block apoptosis, thereby promoting cell survival, Akt has been implicated as a major factor in tumorigenesis in many types of cancer. Akt can be activated by phosphatidylinositol 3-kinase (PI-3K), so the PI-3K antagonists wortmannin and LY294002 may induce apoptotic signals by inhibiting Akt activation ${ }^{[14,37]}$. Recent evidence demonstrates the involvement of the activation of Akt in ischemic tolerance. Nakajima et al showed that Akt was activated in both non-preconditioned and preconditioned animals $1 \mathrm{~h}$ after ischemia, but the activation was long-lasting in the preconditioned rats. They also demonstrated that the preconditioning treatment inhibited the augmentation of neuronal apoptosis in the penumbral region to prevent the spread of infarction, and that the preconditioning-induced neuroprotection was due to the persistent activation of Akt in the penumbra ${ }^{[37]}$. Wick et al demonstrated that neuroprotection by hypoxic preconditioning required sequential activation of vascular endothelial growth factor (VEGF) receptor and $\mathrm{Akt}^{[38]}$. Neurons incubated at $5 \% \mathrm{O}_{2}$ for $9 \mathrm{~h}$ showed increased levels of the VEGF, VEGF receptor-2 (VEGFR-2), phosphorylated Akt/PKB, and ERK1. Incubation with a neutralizing anti-VEGF and anti-VEGFR-2 antibody, wortmannin, or antisense-Akt, reversed the resistance acquired by hypoxic preconditioning. Moreover, inhibition of VEGFR-2 blocked the activation of Akt/PKB, whereas pretreatment with recombinant VEGF also resulted in a hypoxia-resistant phenotype in the absence of hypoxic preconditioning. These data suggested a sequential requirement for
VEGF/VEGFR-2 activation and Akt/PKB phosphorylation for neuronal survival mediated by hypoxic preconditioning, and further implicated Akt/PKB as a major mediator in VEGFdependent neuroprotection.

The PKC family of serine/threonine kinases consists of 10 different isozymes. In the brain and spinal cord, $\mathrm{PKCa}$, PKC $\beta 1, P K C \beta 2, P K C \gamma, P K C \varepsilon, P K C \delta, P K C \eta, P K C \theta$, and PKC乡 mRNA and protein are present and demonstrate unique tissue, cellular, and subcellular localizations. Previous reports suggest that ischemic preconditioning enhances downregulation of cell signaling mediated by $\mathrm{PKC}_{\gamma}$, normalization of calcium homeostasis, and activation of PKC $\delta$ and $\varepsilon^{[39]}$. Recent data demonstrate the key role of the PKC signaling pathway in the context of ischemic preconditioning. Ischemic preconditioning promoted significant increases in the levels of synaptosomal PKC $\varepsilon$ in rat hippocampus. Activation of PKC $\varepsilon$ increased synaptosomal mitochondrial respiration and phosphorylation of mitochondrial respiratory chain proteins ${ }^{[40]}$. Delivery of a PKCE inhibitory peptide abated NMDA-induced preconditioning in cell culture and isolated hippocampal slice models ${ }^{[4]]}$. Correspondingly, delivery of a PKC $\varepsilon$-specific activator peptide reduced damage, as measured with lactate dehydrogenase (LDH) release, when administered before OGD in pure neuronal and mixed neuronal/astrocyte cultures ${ }^{[2]}$. The molecular basis of PKC $\varepsilon$-induced protection is unclear. One mechanism implicates adenosine and $\mathrm{mK}_{\mathrm{ATP}}^{+}$. In ischemic preconditioning, increases in adenosine levels (in addition to opioids) initiate a series of intracellular signaling events via G-protein coupled receptor signaling, leading to activation of phospholipases, production of diacylglycerol (DAG), calcium influx, and PKC activation ${ }^{[43]}$. Multiple studies have now demonstrated that adenosine administration protects neuronal cells against ischemic-type injury via PKC $\varepsilon$. In addition, $\mathrm{PKC} \varepsilon$ activity at the mitochondria may also contribute to regulation of $\mathrm{mK}_{\text {ATP }}^{+}$channels, important for preserving mitochondrial membrane potential, maintaining energy and reducing calcium influx during metabolic challenge ${ }^{[44]}$.

\section{Bcl-2 protein}

The protein Bcl-2 is an anti-apoptotic protein that resides in the outer mitochondrial membranes and the membranes of the endoplasmic reticulum. Overexpression of Bcl-2 is known to block the release of cytochrome $c$, which contributes to a signaling pathway leading to apoptosis. Kato et a ${ }^{[45]}$ showed that two minutes of bilateral carotid artery occlusion in gerbils produced an increase in Bcl-2 at $30 \mathrm{~h}$ and peaked at $96 \mathrm{~h}$, suggesting that the expression of Bcl-2 is involved in ischemic preconditioning. In addition to this, in the study of Shimizu et $a l^{[46]}$, ischemic preconditioning for $20 \mathrm{~min}$ with transient focal ischemia produced ischemic tolerance (attenuated infarction volume). The results of Western blot analysis from tolerant caudate-putamen demonstrated an increase in Bcl-2 expression 3-7 days after preconditioning. Immunocytochemical examination also found that $\mathrm{Bcl}-2$ was expressed in cells with both neuronal and non-neuronal morphology in striatum. Bcl-2 antisense oligodeoxynucleotides (ODNs) treatment 
reduced expression of $\mathrm{Bcl}-2$ in the striatum and blocked the induction of tolerance by preconditioning ischemia. Therefore, Bcl-2 appears to be a major determinant in this model of induced tolerance to focal ischemia. The transcription factors driving the induction of Bcl-2 during tolerance include CREB, which is the Bcl-2 promoter containing a cAMP-responseelement (CRE). As mentioned above, CREB is regulated by NMDA receptor activation, and phosphorylated robustly in the penumbral region of the preconditioned rats ${ }^{[47]}$. Moreover, multiple protein kinases can activate transcription via the CRE, by phosphorylation of CREB, further implicating the interrelation of these different molecular regulatory pathways involved in ischemic preconditioning.

\section{Heat shock proteins (HSPs)}

Although most protein synthesis is inhibited after cerebral ischemia, the remaining active protein synthesis may have an important role in maintaining cell viability. HSPs are molecular chaperones and among the proteins synthesized during ischemia. HSPs are expressed both constitutively (cognate proteins) and under stressful conditions (as inducible forms). In addition to heat shock, a variety of stressful situations including environmental (ultraviolet radiation or heavy metals), pathological (infections or malignancies), or physiological (growth factors or cell differentiation) stimuli induce a marked increase in HSPs synthesis, known as the stress response ${ }^{[48]}$. Inducible HSPs are thought to assist the maintenance of cellular integrity and viability by preventing protein denaturation and improper polypeptide aggregation during exposure to physiochemical insults ${ }^{[49]}$.

HSP70 is the most abundant HSP found in cells. It is expressed constitutively and is only mildly inducible. Many researchers have shown the extensive links between HSP70 overexpression and tolerance in ischemic brain injury. McLaughlin et al have reported an increase in HSP70 expression in an in vivo model of preconditioning ${ }^{[50]}$. Furthermore, HSP70 messenger RNA (mRNA) was compared in the cases of a 15-min ischemia 2 days after sham treatment and a 15-min ischemia 2 days after 10-min preconditioning by Sakurai et $a l^{[51]}$. They reported that HSP70 mRNA in the motor neurons was strong at $8 \mathrm{~h}$ after preconditioning with 10-min ischemia, mild at 1 or 2 days, and not observed at 7 days after preconditioning. Chen et $a l^{[52]}$ reported that thermal preconditioning with $44^{\circ} \mathrm{C}$ body temperature protected cerebellar granule neurons of rats by modulating HSP70 expression. HSP70 mRNA was detected after thermal preconditioning at 30,60, and 90 min and increased gradually with time, whereas HSP70 antisense oligodeoxynucleotides inhibited the protective effects of thermal preconditioning against apoptosis ${ }^{[14,52]}$. All these results implicate that HSP70 is an important player in the preconditioning process.

The underlying mechanisms of HSP70-mediated BIP are not only related to its important functions in protein refolding and transport. Emerging evidence suggests that the HSP70 family is also capable of binding and sequestering activated caspases, such as Apaf and AIF $^{[53]}$. Overexpression of HSP70 inhibits the activation of NF- $\mathrm{kB}$, which is persistently activated during ischemia and appears to promote apoptotic cell death. On the contrary, the deletion of the HSP70 gene notably increases cytochrome $c$ release into the cytoplasm and subsequent caspase-3 activation, thereby exacerbating apoptosis and increasing infarction volume after focal cerebral ischemia ${ }^{[54]}$. Furthermore, HSP70 expression is regulated by transcription factors, the activity of which is increased by ERK phosphorylation ${ }^{[55]}$, a process which has been implicated in preconditioning.

\section{The ubiquitin-proteasome pathway}

There are two protein degradation systems in mammalian cells, the autophagy/lysosomal pathway and the ubiquitinproteasomal pathway. The ubiquitin-proteasomal pathway has been studied in cerebral ischemia and ischemic preconditioning. In rat forebrain ischemia models, transient cerebral ischemia followed by reperfusion leads to delayed selective neuronal death in hippocampal CA1 pyramidal neurons. Under electron microscopy (EM), visible protein aggregates progressively accumulate in some CA1 neurons and accumulation of the aggregates seem to occur primarily in neurons destined to undergo delayed neuronal death after brain ischemia. Further evidence ${ }^{[56,57]}$ showed that the endoplasmic reticulum (ER), mitochondria and cytoplasm all respond to the accumulation of unfolded proteins by compartment-specific signaling pathways to participate in neuronal injury, whereas ubiquintin-proteasome as well as beneficial chaperones function to prevent protein aggregation and assist in protein folding ${ }^{[58,59]}$. Furthermore, by utilizing a rat transient cerebral ischemic preconditioning model, Liu et al found that ischemic preconditioning significantly reduced protein aggregation in CA1 neurons after ischemia. Biochemical analyses revealed that ischemic preconditioning decreased accumulation of ubiquitin-conjugated proteins (ubi-proteins) and reduced free ubiquitin depletion after brain ischemia. Ischemic preconditioning also reduced redistribution of heat shock cognate protein 70 and Hdj1 (HSP40) from cytosolic fraction to protein aggregate-containing fraction after brain ischemia ${ }^{[60]}$.

Indeed, the proteasome comprises multiple protein subunits and degrades cytosolic proteins as well as misfolded proteins that fail to pass protein quality control in the $\mathrm{ER}^{[61]}$. Misfolded proteins in the ER are recognized by ER-specific E3 ligases that mediate polyubiquitination of the misfolded protein on the cytosolic side of the ER and are subsequently degraded by the proteasome ${ }^{[62]}$. Proteasome degradation of ubi-proteins is strictly ATP-dependent. Ubi-proteins serve as signals to activate heat shock transcription factors to induce expression of molecular chaperones ${ }^{[63]}$, which then shield hydrophobic surfaces of proteins in non-native states, thereby blocking their aggregation. Major cellular chaperones are ATPases and assist protein folding through numerous cycles of binding and release of unfolded protein substrates by hydrolysis of ATP. Therefore, ubiquitin-proteasome and molecular chaperons may be concerned with the neuroprotection of ischemic preconditioning and may together prevent protein aggregation in lethal ischemia. 


\section{Hypoxia-inducible factor (HIF)}

Arthur et al developed two in vitro models of ischemia/reperfusion: OGD, in which neuronal cell death was predominantly by necrosis (necrotic model) and programmed cell death (PCD model). Hypoxic preconditioning $24 \mathrm{~h}$ prior to OGD significantly reduced cell death from $83 \%$ to $22 \%$ in the necrotic model and $68 \%$ to $11 \%$ in the PCD model ${ }^{[64]}$. In this IPC model, the activity of the antioxidant enzymes glutathione peroxidase, glutathione reductase, and Mn superoxide dismutase were significantly increased, whereas superoxide and hydrogen peroxide concentrations following OGD were significantly lower in the IPC group. Furthermore, cytochrome $c$ release from mitochondria to the cytosol was suppressed in the ischemia tolerance-induced hippocampal CA1 region ${ }^{[65]}$ Recent reports suggested that one mechanism of preconditioning probably involves hypoxia-inducible factor-1alpha (HIF1alpha $)^{[66,67]}$. HIF-1alpha is a transcription factor that binds with a second protein (HIF-1beta) in the nucleus to promote elements in hypoxia-responsive target genes during hypoxia. This binding causes upregulation of HIF target genes, including VEGF, erythropoietin, iNOS, glucose transporter-1, glycolytic enzymes, and many other genes that protect the brain against ischemia $24 \mathrm{~h}$ later. Hypoxia preconditioning can be mimicked by iron chelators like desferrioxamine and transition metals like cobalt chloride that inhibit prolyl hydroxylases, increase HIF-1alpha levels in the brain, and produce protection of the brain against combined hypoxia-ischemia 24 h later.

\section{Nitric oxide/reactive oxygen species and neuroprotection Nitric oxide}

Gidday and colleagues demonstrated in 1999 that NO is involved in the development of hypoxic and ischemic tolerance ${ }^{[68]}$. They showed that NO production and activity were critical to the induction of ischemic tolerance and that endothelial nitric oxide synthase (eNOS), not neuronal nitric oxide synthase (nNOS) or inducible nitric oxide synthase (iNOS), was the isoform responsible for producing ischemic tolerance. In the study by Sunghee Cho and his colleagues, both inhibiting nitric oxide synthase (NOS) and scavenging NO during preconditioning significantly attenuated the induced ischemic tolerance, however, neither eNOS nor nNOS knockout mice demonstrated altered ischemic preconditioning $^{[14,69,70]}$. Meanwhile, preconditioning by volatile anesthetics also appears to involve the NO pathway. Kapinya and coworkers showed that tolerance against ischemic neuronal injury in rats can be induced by volatile anesthetics and that this effect is dependent on iNOS ${ }^{[14,71]}$.

The exact mechanisms responsible for NO-induced induction of ischemic tolerance are not clear. Up-regulation of eNOS appears to be triggered via the PI3-K pathway, and both eNOS and PI3-K contribute to ischemic tolerance in the CA1 neurons of gerbil hippocampus ${ }^{[72]}$. Furthermore, NO appears to activate Ras and then the ERK cascade, which is also known as the microtubule-associated or MAPK pathway ${ }^{[14]}$. Ras is a G protein, a regulatory GTP hydrolase that cycles between two conformations - an activated or inactivated form. Ras is activated by mitogenic signals as well as autocatalytically through a feedback mechanism. Ras activates a number of pathways, but the most important seems to be MAPK, which transmits signals downstream to other protein kinases and gene regulatory proteins. Gonzalez-Zulueta and collaborators reported the essential role of $\mathrm{NO}$ activation in neuronal ischemic preconditioning ${ }^{[3]}$, and also found that preconditioning induced Ras activation in an NMDA receptor- and NO-dependent, but cGMP-independent manner. They also demonstrated that Ras activity was necessary and sufficient for ischemic tolerance induction in neurons. Pharmacological inhibition of Ras by farnesyl protein transferase inhibitor III, as well as a dominant Ras negative mutant, blocked preconditioning, whereas a constitutively active form of Ras promoted the induction of ischemic tolerance against lethal insults. These studies suggest that Ras might be the downstream factor of NO-mediated neuroprotection in ischemic preconditioning ${ }^{[14]}$.

\section{Reactive oxygen species (ROS)}

Reactive oxygen species (ROS) are traditionally recognized as important intracellular signals implicated in myocardial ischemia/reperfusion injury ${ }^{[74,75]}$. Mitochondria are the main source of ROS, and ROS are mainly regarded as toxic byproducts of metabolism with the potential to cause damage to lipids, proteins, and $\mathrm{DNA}^{[76]}$. It has been demonstrated that endogenous free radicals (the ROS product) can cause cellular calcium loading with inhibition of the sarcoplasmic reticulum calcium ATPase and inhibition of the sodium potassium ATPase, leading to sodium mediated calcium gain. Meanwhile, oxygen radicals are also responsible for lipid peroxidation, resulting in cell membrane breakdown and cell swelling ${ }^{[77]}$. Although mammalian cells express endogenous free radical scavenging enzymes ${ }^{[74]}$, such as superoxide dismutase (which reduces $\mathrm{O}_{2}^{-} \cdot$ to $\mathrm{H}_{2} \mathrm{O}_{2}$ ), catalase, and glutathione peroxidase (which reduces $\mathrm{H}_{2} \mathrm{O}_{2}$ to $\mathrm{H}_{2} \mathrm{O}$ ), these antioxidative defenses are overwhelmed after ischemia and reperfusion.

However, recent studies ${ }^{[5,78]}$ indicate that oxygen free radicals also have an important role in triggering the signal transduction pathways in ischemic preconditioning. Some oxygen free radicals such as hydroxyl radicals $(\cdot \mathrm{OH})$ may be involved in ischemic preconditioning induction ${ }^{[79]}$. The relationship between oxygen free radicals and ischemic preconditioning was first suggested by Murry and colleagues ${ }^{[80]}$, who showed that administration of oxygen radical scavengers during the first reperfusion period could block the beneficial effect of IP on infarct size in dogs. They therefore proposed that the generation of a low amount of oxygen free radicals during a short ischemic episode is not sufficient to cause cell necrosis, but enough to modify cellular activity and induce preconditioning effects. Therefore, low levels of oxygen radicals and oxidants are normally formed in cells and play an important part in cellular homeostasis ${ }^{[7]}$

\section{Autophagy/lysosomal pathway and neuroprotection}

Recently, the roles of autophagy and the lysosomal pathway in 
cerebral ischemia have attracted intensive attention. Studies have reported the activation of autophagy following ischemic insults, but the contribution of autophagy to neuronal death/ survival is still under debate ${ }^{[81-85]}$. In an earlier study, we found profound activation of autophagy and lysosomes after permanent middle cerebral artery occlusion (pMCAO) and an autophagic mechanism may contribute to ischemic neuronal injury $^{[86]}$.

However, autophagy is a double-edged sword ${ }^{[87]}$. Although massive autophagy is associated with cell death through excessive self-digestion and degradation of cellular constitutions, activation of autophagy can also protect the neurons by degrading unfolded proteins and damaged organelles. A study carried out in primary cultured cardiomyocytes ${ }^{[88]}$ showed that inhibition of autophagy with 3-methyladenine (3-MA, an autophagy inhibitor) during anoxia-reoxygenation caused an increase in the number of necrotic cells and a decrease of the living cell population. Thus, the process of autophagy during anoxia-reoxygenation was proposed to provide some protective effects. In addition, a recent in vitro study in PC12 cells also showed that autophagy was associated with neuroprotective effects induced by IPC ${ }^{[89]}$. Inhibition of autophagy, especially during reperfusion or lethal oxygen-glucose deprivation periods, ameliorated the neuroprotective effects of IPC, and also attenuated HSP70 upregulation induced by IPC. Furthermore, our recent observations showed that autophagy was also induced in a rat ischemic preconditioning model. Pretreatment with 3-MA before the onset of ischemic preconditioning nearly completely suppressed the neuroprotective effects of preconditioning. These results strongly supported the hypothesis that induction of autophagy in ischemic preconditioning may play a part in protecting against the sequential lethal cerebral ischemia (Sheng et al, manuscript submitted).

\section{Potential clinical applications of preconditioning}

Since the first description of ischemic preconditioning, it has been demonstrated experimentally that endotoxin, tumor necrosis factor-a, and metabolic inhibitor 3-nitro-propionic acid can mimic ischemic preconditioning and induce BIP in the brain. Unfortunately, the clinical use of these substances is unacceptable because of their toxicity or other side effects.

However, preconditioning promises practical usefulness for vascular neurosurgery, cardiovascular surgery and possibly also in the management of brain tumors and trauma. Chan and his team applied ischemic preconditioning for cerebral aneurysm surgery ${ }^{[90]}$. They evaluated the effects of ischemic preconditioning produced by 2 min proximal temporary artery occlusion on brain tissue gases and acidity during clipping of cerebral aneurysms. Twelve patients with aneurismal subarachnoid hemorrhage were recruited. In patients assigned to the preconditioning group, the proximal artery was occluded initially for $2 \mathrm{~min}$ and was allowed to reperfuse for $30 \mathrm{~min}$. All patients underwent cerebral artery occlusion for clipping of the aneurysm. Baseline brain tissue gases and $\mathrm{pH}$ were similar between groups. However, following artery occlusion, the declines in oxygen tension and $\mathrm{pH}$ were significantly slower in the preconditioning group compared with the routine care group. These results suggested that a brief occlusion of the proximal artery may be a simple maneuver for brain protection during complex cerebrovascular surgery. Moreover, hyperbaric oxygen $\left(\mathrm{O}_{2}\right)$ is also able to mimic ischemic preconditioning and induce neuroprotection against ischemic injury in animal brains and spinal cords. In addition, some easily applied methods of preconditioning such as the application of isoflurane can also be introduced conveniently into the practice for operation of neurosurgery.

On the other hand, certain pharmacologic agents have been shown to be as effective as ischemic preconditioning in achieving their protective effects on cerebral ischemia, and are described as pharmacological preconditioning ${ }^{[91]}$. Those agents that affect key proteins involved in the protective signaling pathways can potentially be used as tolerance-producing drugs. Recently, research from our laboratory ${ }^{[92,93]}$ showed that pretreatment with prostaglandin A1 (PGA1) or PGE1 and lithium significantly reduced infarct volume, neurological deficits and brain edema in MCAO rats, and their combination (PGA1+lithium, PGE1+lithium) exert greater neuroprotection in the MCAO model. Importantly, all these drugs significantly enhanced the expression of HSP70 in the ischemic striatum, which is widely accepted to be involved in ischemic preconditioning. Moreover, lithium also activates autophagy activity ${ }^{[94]}$. Actually, the selective autophagy activator rapamycin can mimic the biological effects of preconditioning (Sheng et al, manuscript submitted). We thus speculate that drugs inducing HSPs and activating autophagy may be used for pharmacological preconditioning. The possible cumulative neuroprotection by pharmacological preconditioning should be studied systematically.

\section{Conclusion}

Current knowledge suggests that the concept of preconditioning can help to improve neuronal survival after temporary critical ischemia. Although the mechanisms behind the formation of protection are still largely unknown, significant progress has been made toward identifying some of the major molecules involved (Figure 1). Molecules and proteins that are important for the development of ischemic tolerance in the brain are potential targets for the development of new treatments for ischemia. One strategy to identify such targets is to look at factors that have already been determined to be important for tolerance in other tissues. As mentioned previously, ischemic tolerance was first identified in the heart and has been studied more extensively in the heart than in the brain. It is likely that some of the processes essential for the development of tolerance are the same in both tissues. Newly identified targets in the heart should therefore be considered as being potentially important in the brain, and vice versa. Another strategy to identify new targets is to look at molecules and proteins that interact with currently known targets. If one component of a particular pathway is known to be important for the development of tolerance, the other components of that 


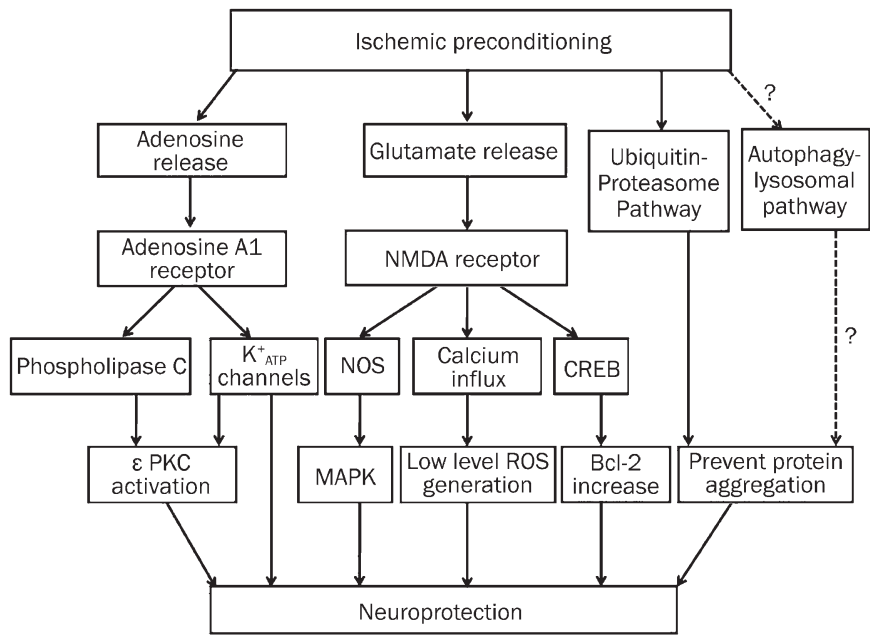

Figure 1. Simplified scheme depicting some basic signaling pathways involved in the neuroprotection of brain ischemic preconditioning. Triggering pathways include activation of the NMDA and adenosine A1 receptors which in turn are involved in activating some intracellular signaling pathways such as mitogen-activated protein kinases (MAPKs), protein kinase $\mathrm{C}(\mathrm{PKC}), \mathrm{bcl}-2$, heat shock proteins (HSPs), ubiquitin-proteasome pathway and autophagic-lysosomal pathway. NMDA receptor: $N$-methyl- $D$-aspartate receptor; NOS: nitric oxide synthase; CREB: cyclic AMP responsive element binding protein; ROS: reactive oxygen species.

pathway might be important as well. Of course, there may be novel factors involved in tolerance that cannot be detected by these strategies and that will require broader studies. Once the processes involved in preconditioning are more fully understood, the potential benefits for prevention and treatment of brain damage due to ischemia are substantial.

\section{Acknowledgements}

This work was supported by grants from the National Natural Science Foundation of China (№ 30801391, 30470587), the Natural Science Foundation of Jiangsu Province (№ BK 2007548), and Suzhou Social Progress Foundation SS0729.

\section{References}

1 Dirnagl U, Simon RP, Hallenbeck JM. Ischemic tolerance and endogenous neuroprotection. Trends Neurosci 2003; 26: 248-54.

2 Yanamoto H, Hashimoto N, Nagata I, Kikuchi H. Infarct tolerance against temporary focal ischemia following spreading depression in rat brain. Brain Res 1998; 784: 239-49.

3 Hsu JC, Lee YS, Chang CN, Ling EA, Lan CT. Sleep deprivation prior to transient global cerebral ischemia attenuates glial reaction in the rat hippocampal formation. Brain Res 2003; 984: 170-81.

4 Yu ZF, Mattson MP. Dietary restriction and 2-deoxyglucose administration reduce focal ischemic brain damage and improve behavioral outcome: evidence for a preconditioning mechanism. J Neurosci Res 1999; 57: 830-9.

5 Wiegand F, Liao W, Busch C, Castell S, Knapp F. Respiratory chain inhibition induces tolerance to focal cerebral ischemia. J Cereb Blood Flow Metab 1999; 19: 1229-37

6 Zemke D, Smith JL, Reeves MJ, Majid A. Ischemia and ischemic tolerance in the brain. NeuroToxicology 2004; 25: 895-904.

7 Mehta SL, Manhas N, Raghubir R. Molecular targets in cerebral ischemia for developing novel therapeutics. Brain Res Rev 2007; 54: 34-66.

8 Jiang $\mathrm{X}$, Zhu D, Okagaki P, Lipsky R, Wu X. N-methyl-D-aspartate and TrkB receptor activation in cerebellar granule cells: an in vitro model of preconditioning to stimulate intrinsic survival pathways in neurons. Ann NY Acad Sci 2003; 993: 134-45.

9 Bond A, Lodge D, Hicks CA, Ward MA, O'Neill MJ. NMDA receptor antagonism, but not AMPA receptor antagonism attenuates induced ischemic tolerance in the gerbil hippocampus. Eur J Pharmacol 1999; 380: 91-9.

10 Grabb MC, Choi DW. Ischemic tolerance in murine cortical cell culture: critical role for NMDA receptors. J Neurosci 1999; 19: 1657 62.

11 Shimazaki K, Nakamura T, Nakamura K, Oguro K, Masuzawa T, Kudo $Y$, et al. Reduced calcium elevation in hippocampal CA1 neurons of ischemia-tolerant gerbils. Neuroreport 1998; 9: 1875-8.

12 Mattson MP, Goodman Y, Luo H, Fu W, Furukawa K. Activation of NF$K B$ protects hippocampal neurons against oxidative stress-induced apoptosis: Evidence for induction of manganese superoxide dismutase and suppression of peroxynitrite production and protein tyrosine nitration. J Neurosci Res 1997; 49: 681-97.

13 Soriano FX, Papadia S, Hofmann F, Hardingham NR. Preconditioning doses of NMDA promote neuroprotection by enhancing neuronal excitability. J Neurosci 2006; 26: 4509-18.

14 Steiger HJ, Hänggi D. Ischemic preconditioning of the brain, mechanisms and applications. Acta Neurochir (Wien) 2007; 149: 1-10.

15 Hu BR, Fux CM, Martone ME, Zivin JA, Ellisman MH. Persistent phosphorylation of cyclic AMP responsive element-binding protein and activating transcription factor-2 transcription factors following transient cerebral ischemia in rat brain. Neuroscience 1999; 89: 437-52.

16 Nakajima T, Iwabuchi S, Miyazaki H, Okuma Y, Inanami O, Kuwabara M, et al. Relationship between the activation of cyclic AMP responsive element binding protein and ischemic tolerance in the penumbra region of rat cerebral cortex. Neurosci Lett 2002; 331: 13-6.

17 Perez-Pinzon MA. Mechanisms of neuroprotection during ischemic preconditioning: Lessons from anoxic tolerance. Comp Biochem Physiol A Mol Integr Physiol 2007; 147: 291-9.

18 Dave KR, Lange-Asschenfeldt C, Raval AP, Prado R, Busto R, Saul I,et al. Ischemic preconditioning ameliorates excitotoxicity by shifting glutamate/gamma-aminobutyric acid release and biosynthesis. J Neurosci Res 2005; 82: 665-73.

19 Sommer C, Fahrner A, Kiessling M. Postischemic neuroprotection in the ischemia-tolerant state gerbil hippocampus is associated with increased ligand binding to inhibitory GABA(A) receptors. Acta Neuropathol (Berl) 2003; 105: 197-202.

20 Obrenovitch TP. Molecular physiology of preconditioning-induced brain tolerance to ischemia. Physiol Rev 2008; 88: 211-47.

21 Kristian T, Siesjo BK. Calcium in ischemic cell death. Stroke 1998; 29 : 705-18.

22 Zhou AM, Li WB, Li QJ, Liu HQ, Feng RF, Zhao HG. A short cerebral ischemic preconditioning up-regulates adenosine receptors in the hippocampal CA1 region of rats. Neurosci Res 2004; 48: 397-404.

23 Liu Y, Xiong L, Chen S, Wang Q. Isoflurane tolerance against focal cerebral ischemia is attenuated by adenosine A1 receptor antagonists. Can J Anaesth 2006; 53: 194-201.

24 Pérez-Pinzón MA, Born JG. Rapid preconditioning neuroprotection following anoxia in hippocampal slices: role of $\mathrm{K}_{\text {ATP }}^{+}$channel and protein kinase C. Neuroscience 1999; 89: 453-9.

25 Schultz JEJ, Qian YZ, Gross GJ, Kukreja RC. The ischemia selective 
K-ATP channel antagonist, 5-hydroxydecanoate, blocks ischemic preconditioning in the rat heart. J Mol Cell Cardiol 1997; 29: 1055-60.

26 Zhang Y, Wu YX, Hao YB, Dun Y, Yang SP. Role of endogenous opioid peptidesin protection of ischemic preconditioning in rat small intestine. Life Sci 2001; 68: 1013-9.

27 Zhao P, Huang Y, Zuo Z. Opioid preconditioning induces opioid receptor-dependent delayed neuroprotection against ischemia in rats. Neuropathol Exp Neurol 2006; 65: 945-52.

28 Kariko K, Weissmann D, Welsh FA. Inhibition of toll-like receptor and cytokine signaling - a unifying theme in ischemic tolerance. J Cereb Blood Flow Metab 2004; 24: 1288-304.

29 Nawashiro H, Tasaki K, Ruetzler CA, Hallenbeck JM. TNF-alpha pretreatment induces protective effects against focal cerebral ischemia in mice. J Cereb Blood Flow Metab 1997; 17: 483-90.

30 Liu J, Ginis I, Spatz M, Hallenbeck JM. Hypoxic preconditioning protects cultured neurons against hypoxic stress via TNF-alpha and ceramide. Am J Physiol Cell Physiol 2000; 278: C144-53.

31 Ohtsuki T, Ruetzler CA, Tasaki K, Hallenbeck JM. Interleukin-1 mediates induction of tolerance to global ischemia in gerbil hippocampal CA1 neurons. J Cerb Blood Flow Metab 1996; 16: 1137-42.

32 Huang J, Upadhyay UM, Tamargo RJ. Inflammation in stroke and focal cerebral ischemia. Surg Neurol 2006; 66: 232-45.

33 Nozaki K, Nishimura M, Hashimoto N. Mitogen-activated protein kinases and cerebral ischemia. Mol Neurobiol 2001; 23: 1-19.

34 Mehta SL, Manhas N, Raghubir R. Molecular targets in cerebral ischemia for developing novel therapeutics. Brain Res Rev 2007; 54 : 34-66.

35 Zheng S, Zuo Z. Isoflurane preconditioning reduces purkinje cell death in an in vitro model of rat cerebellar ischemia. Neuroscience 2003; 118: 99-106.

36 Zheng S, Zuo Z. Isoflurane preconditioning induces neuroprotection against ischemia via activation of p38 mitogen-activated protein kinases. Mol Pharmacol 2004; 65: 1172-80.

37 Nakajima T, Iwabuchi S, Miyazaki H, Okuma Y, Kuwabara M, Nomura $\mathrm{Y}$, et al. Preconditioning prevents ischemia-induced neuronal death through persistent Akt activation in the penumbra region of the rat brain. J Vet Med Sci 2004; 66: 521-7.

38 Wick A, Wick W, Waltenberger J, Weller M, Dichgans J, Schulz JB, et al. Neuroprotection by hypoxic preconditioning requires sequential activation of vascular endothelial growth factor receptor and Akt. J Neurosci 2002; 22: 64-71.

39 Bright R, Mochly-Rosen D. The role of protein kinase C in cerebral ischemic and reperfusion injury. Stroke 2005; 36: 2781-90.

40 Dave KR, DeFazio RA, Raval AP, Torraco A, Saul I, Barriento A, et al. Ischemic preconditioning targets the respiration of synaptic mitochondria via protein kinase CE. J Neurosci 2008; 28: 4172-82.

41 Raval AP, Dave KR, Mochly-Rosen D, Sick TJ, Pérez-Pinzón MA. EpsiIon $\mathrm{PKC}$ is required for the induction of tolerance by ischemic and NMDA-mediated preconditioning in the organotypic hippocampal slice. J Neurosci 2003; 23: 384-91.

42 Wang J, Bright R, Mochly-Rosen D, Giffard RG. Cell-specific role for epsilon- and betal-protein kinase $\mathrm{C}$ isozymes in protecting cortical neurons and astrocytes from ischemia-like injury. Neuropharmacology $2004 ; 47: 136-45$.

43 Di-Capua N, Sperling O, Zoref-Shani E. Protein kinase C-epsilon is involved in the adenosine-activated signal transduction pathway conferring protection against ischemia-reperfusion injury in primary rat neuronal cultures. J Neurochem 2003; 84: 409-12.

44 Raval AP, Dave KR, DeFazio RA, Perez-Pinzon MA. $\varepsilon$ PKC phosphorylates the mitochondrial $\mathrm{K}_{\text {ATP }}^{+}$channel during induction of ischemic preconditioning in the rat hippocampus. Brain Res 2007; 1184:
345-53.

45 Kato H, Liu Y, Araki T, Kogure K. Temporal profile of the effects of pretreatment with brief cerebral ischemia on the neuronal damage following secondary ischemic insult in the gerbil: cumulative damage and protective effects. Brain Res 1991; 553: 238-42.

46 Shimizu S, Nagayama T, Jin KL, Zhu L, Loeffer JE, Watkins SC, et al. $\mathrm{Bcl}-2$ antisense treatment prevents induction of tolerance to focal ischemia in the rat brain. J Cereb Blood Flow Metab 2001; 21: $233-$ 43.

47 Nakajima T, Iwabuchi S, Miyazaki H, Okuma Y, Inanami O, Kuwabara M, et al. Relationship between the activation of cyclic AMP responsive element binding protein and ischemic tolerance in the penumbra region of rat cerebral cortex. Neurosci Lett 2002; 331: 13-6.

48 Tsan MF, Gao B. Cytokine function of heat shock proteins. Am J Physiol Cell Physiol 2004; 286: 739-44.

49 Ravati A, Ahlemeyer B, Becker A, Klumpp S, Krieglstein J. Preconditioning-induced neuroprotection is mediated by reactive oxygen species and activation of the transcription factor nuclear factorkappaB. J Neurochem 2001; 78: 909-19.

50 McLaughlin B, Hartnett KA, Erhardt JA, Legos JJ, White RF, Barone $\mathrm{FC}$, et al. Caspase 3 activation is essential for neuroprotection in preconditioning. Proc Natl Acad Sci USA 2003; 100: 715-20.

51 Sakurai M, Hayashi T, Abe K, Aoki M, Sadahiro M, Tabayashi K, et al. Enhancement of heat shock protein expression after transient ischemia in the preconditioned spinal cord of rabbits. J Vasc Surg 1998; 27: 720-5.

52 Chen L, Su XW, Qiu PX, Huang YJ, Yan GM. Thermal preconditioning protected cerebellar granule neurons of rats by modulating HSP70 expression. Acta Pharmacol Sin 2004; 25: 458-61.

53 Yenari MA, Giffard RG, Sapolsky RM, Steinberg GK. The neuroprotective potential of heat shock protein 70 (HSP70). Mol Med Today 1999; 5: 525-31.

54 Lee SH, Kwon HM, Kim YJ, Lee KM, Kim M, Yoon BW. Effects of HSP70.1 gene knockout on the mitochondrial apoptotic pathway after focal cerebral ischemia. Stroke 2004; 35: 2195-9.

55 Minet E, Arnould T, Michel G, Roland I, Mottet D, Raes M, et al. ERK activation upon hypoxia: involvement in HIF-1 activation. FEBS Lett 2000; 46: 53-8.

56 Yoneda T, Benedetti C, Urano F, Clark SG, Harding HP, Ron D. Compartment-specific perturbation of protein handling activates genes encoding mitochondrial chaperones. J Cell Sci 2004; 117: 4055-66.

57 DeGracia DJ, Hu BR. Irreversible translation arrest in the reperfused brain. J Cereb Blood Flow Metab 2007; 27: 875-93.

58 Hebert DN, Molinari M. In and out of the ER: protein folding, quality control, degradation, and related human diseases. Physiol Rev 2007; 8: 1377-408.

59 Ghaemmaghami S, Huh WK, Bower K, Howson RW, Belle A, Dephoure $\mathrm{N}$, et al. Global analysis of protein expression in yeast. Nature 2003; 425: 737-41.

60 Liu C, Chen S, Kamme F, Hu BR. Ischemic preconditioning prevents protein aggregation after transient cerebral ischemia. Neuroscience 2005; 134: 69-80.

$61 \mathrm{Wu}$ JC. Liang ZQ. Qin ZH. Quality control system of the endoplasmic reticulum and related diseases. Acta Biochem Biophys Sin 2006; 38 : 219-26.

62 Chen JJ, Lin F, Qin ZH. The roles of the proteasome pathway in signal transduction and neurodegenerative diseases. Neurosci Bull 2008; 24: 183-94.

63 Voellmy R. Transcriptional regulation of the metazoan stress protein response. Prog Nucleic Acid Res Mol Biol 2004; 78: 143-85. 
64 Arthur PG, Lim SC, Meloni BP, Munns SE, Chan A, Knuckey NW. The protective effect of hypoxic preconditioning on cortical neuronal cultures is associated with increases in the activity of several antioxidant enzymes. Brain Res 2004; 1017: 146-54.

65 Nakatsuka H, Ohta S, Tanaka J, Toku K, Kumon Y, Maeda N, et al. Cytochrome $c$ release from mitochondria to the cytosol was suppressed in the ischemia-tolerance-induced hippocampal CA1 region after 5-min forebrain ischemia in gerbils. Neurosci Lett 2000; 278 : 53-6.

66 Ran R, Xu H, Lu A, Bernaudin M. Hypoxia preconditioning in the brain. Dev Neurosci 2005; 27: 87-92.

67 Gu GJ, Li YP, Peng ZY, Xu JJ, Kang ZM, Xu WG, et al. Mechanism of ischemic tolerance induced by hyperbaric oxygen preconditioning involves upregulation of hypoxia-inducible factor-1and erythropoietin in rats. J Appl Physiol 2008; 104: 1185-91.

68 Gidday JM, Shah AR, Maceren RG, Wang Q, Pelligrino DA, Holtzman $\mathrm{DM}$, et al. Nitric oxide mediates cerebral ischemic tolerance in a neonatal rat model of hypoxic preconditioning. J Cereb Blood Flow Metab 1999; 19: 331-40.

69 Atochin DN, Clark J, Demchenko IT, Moskowitz MA, Huang PL. Rapid cerebral ischemic preconditioning in mice deficient in endothelial and neuronal nitric oxide synthases. Stroke 2003; 34: 1299-303.

70 Cho S, Park EM, Zhou P, Frys K, Ross ME, ladecola C. Obligatory role of inducible nitric oxide synthase in ischemic preconditioning. J Cereb Blood Flow Metab 2005; 25: 493-501.

71 Kapinya KJ, Prass K, Dirnagl U. Isoflurane induced prolonged protection against cerebral ischemia in mice: a redox sensitive mechanism? Neuroreport 2002; 13: 1431-5.

72 Hashiguchi A, Yano S, Morioka M, Hamada J, Ushio Y, Takeuchi Y, et al. Up-regulation of endothelial nitric oxide synthase via phosphatidylinositol 3-kinase pathway contributes to ischemic tolerance in the CA1 subfield of gerbil hippocampus. J Cereb Blood Flow Metab 2004; 24 : 271-9.

73 Gonzalez-Zulueta M, Feldman AB, Klesse LJ, Kalb RG, Dillman JF, Parada L, et al. Requirement for nitric oxide activation of p21(ras)/ extracellular regulated kinase in neuronal ischemic preconditioning. Proc Natl Acad Sci USA 2000; 9: 436-41.

74 Dhalla NS, Elmoselhi AB, Hata T, Makino N. Status of myocardial antioxidants in ischemia-reperfusion injury. Cardiovasc Res 2000; 47; 446-56.

75 Jin ZQ, Zhou HZ, Cecchini G, Gray MO, Karliner JS. MnSOD in mouse heart: acute responses to ischemic preconditioning and ischemiareperfusion injury. Am J Physiol Heart Circ Physiol 2005; 288: 298694.

76 Thannickal VJ, Fanburg BL. Reactive oxygen species in cell signaling. Am J Physiol Lung Cell Mol Physiol 2000; 279: 1005-28.

77 Zweier JL, Talukder MAH. The role of oxidants and free radicals in reperfusion injury. Cardiovas Res 2006; 70: 181-90.

78 Wada K, Miyazawa T, Nomura N, Tsuzuki N, Nawashiro H, Shima K, et al. Preferential conditions for and possible mechanisms of induction of ischemic tolerance by repeated hyperbaric oxygenation in gerbil hippocampus. Neurosurgery 2001; 49: 160-7.

79 Rauca C, Zerbe R, Jantze H, Krug M. The important of free hydroxyl radicals to hypoxia preconditioning. Brain Res 2000; 868: 147-9.

80 Murry CE, Richard VJ, Jennings RB, Reimer KA. Preconditioning with isehemia: is the protective effect mediated by free radical induced myocardial stunning? Circulation 1988; 78: 77.

81 Adhami F, Liao G, Morozov YM, Schloemer A, Schmithorst VJ, Lorenz $J N$, et al. Cerebral ischemia-hypoxia induces intravascular coagulation and autophagy. Am J Pathol 2006; 169: 566-83.

82 Carloni S, Buonocore G, Balduini W. Protective role of autophagy in neonatal hypoxia-ischemia induced brain injury. Neurobiol Dis 2008; 32: 329-39.

83 Koike M, Shibata M, Tadakoshi M, Gotoh K, Komatsu M, Waguri S, et al. Inhibition of autophagy prevents hippocampal pyramidal neuron death after hypoxic-ischemic injury. Am J Pathol 2008; 172: 454-69.

84 Rami A, Langhagen A, Steiger S. Focal cerebral ischemia induces upregulation of Beclin 1 and autophagy-like cell death. Neurobiol Dis 2008; 29: 132-41.

85 Balduini W, Carloni S, Buonocore G. Autophagy in hypoxia-ischemia induced brain injury: Evidence and speculations. Autophagy 2009; 5: 221-3.

86 Wen YD, Sheng R, Zhang LS, Han R, Zhang X, Zhang XD, et al. Neuronal injury in rat model of permanent focal cerebral ischemia is associated with activation of autophagic and lysosomal pathways. Autophagy 2008; 4: 762-9.

87 Shintani T, Klionsky DJ. Autophagy in health and disease: a doubleedged sword. Science 2004; 306: 990-5.

88 Dosenko VE, Nagibin VS, Tumanovska LV, Moibenko AA. Protective effect of autophagy in anoxia-reoxygenation of isolated cardiomyocyte? Autophagy 2006; 2: 305-6.

89 Park HK, Chu K, Jung KH, Lee ST, Bahn JJ, Kim M, et al. Autophagy is involved in the ischemic preconditioning. Neurosci Lett 2009; 451: 16-9.

90 Chan MT, Boet R, Ng SC, Poon WS, Gin T. Effect of ischemic preconditioning on brain tissue gases and $\mathrm{pH}$ during temporary cerebral artery occlusion. Acta Neurochir 2005; 95: 93-96.

91 Ratan RR, Siddiq A, Aminova L, Lange PS, Langley B, Ayoub I, et al. Translation of ischemic preconditioning to the patient: prolyl hydroxylase inhibition and hypoxia inducible factor-1 as novel targets for stroke therapy. Stroke 2004; 35: 2687-89.

$92 \mathrm{Xu} \mathrm{XH}$, Zhang HL, Han R, Gu ZL, Qin ZH. Enhancement of neuroprotection and heat shock protein induction by combined prostaglandin A1 and lithium in rodent models of focal ischemia. Brain Res 2006; 1102: 154-62.

93 Han R, Gao B, Sheng R, Zhang LS, Zhang HL, Gu ZL, et al. Synergistic effects of prostaglandin $\mathrm{E} 1$ and lithium in a rat model of cerebral ischemia. Acta Pharmacol Sin 2008; 29: 1141-9.

94 Sarkar S, Floto RA, Berger Z, Imarisio S, Cordenier A, Pasco M, et al. Lithium induces autophagy by inhibiting inositol monophosphatase. J Cell Biol 2005; 170: 1101-11. 